\title{
A Banach Space Regularization Approach for Multifrequency Microwave Imaging
}

\author{
Claudio Estatico, ${ }^{1}$ Alessandro Fedeli, ${ }^{2}$ Matteo Pastorino, ${ }^{2}$ and Andrea Randazzo ${ }^{2}$ \\ ${ }^{1}$ Department of Mathematics, University of Genoa, Via Dodecaneso 35, 16146 Genova, Italy \\ ${ }^{2}$ Department of Electrical, Electronic, Telecommunications Engineering, and Naval Architecture, University of Genoa, \\ Via Opera Pia 11A, 16145 Genova, Italy
}

Correspondence should be addressed to Andrea Randazzo; andrea.randazzo@unige.it

Received 15 October 2015; Accepted 24 February 2016

Academic Editor: Ahmed T. Mobashsher

Copyright (C) 2016 Claudio Estatico et al. This is an open access article distributed under the Creative Commons Attribution License, which permits unrestricted use, distribution, and reproduction in any medium, provided the original work is properly cited.

\begin{abstract}
A method for microwave imaging of dielectric targets is proposed. It is based on a tomographic approach in which the field scattered by an unknown target (and collected in a proper observation domain) is inverted by using an inexact-Newton method developed in $L^{p}$ Banach spaces. In particular, the extension of the approach to multifrequency data processing is reported. The mathematical formulation of the new method is described and the results of numerical simulations are reported and discussed, analyzing the behavior of the multifrequency processing technique combined with the Banach spaces reconstruction method.
\end{abstract}

\section{Introduction}

The regularization of ill-posed problems in $L^{p}$ Banach spaces exhibits several advantages over the corresponding classical regularization in Hilbert spaces [1-3]. A significant example is microwave imaging (MI). MI is a well-known technique in which an unknown target is inspected by using an electromagnetic radiation [4]. When illuminated by an incident field, the target scatters the incident radiation depending on its physical properties, geometrical shape, and dimensions. For dielectric targets with dimensions comparable with the wavelength of the incident waves, the scattering phenomena are quite complicated [5]. However, the scattered electric field can be collected around the target and the properties of the object can be retrieved by inverting the equation governing the electromagnetic scattering problem. Several approaches to solve this inverse problem with qualitative [68] or quantitative techniques [9-11] have been reported in the scientific literature, with reference to both two- and threedimensional configurations [12-14]. Among these methods, it has been proved in [15] that, exploiting the properties of the norms in the $L^{p}$ Banach spaces, it is possible to obtain better reconstructions than developing the corresponding approach in Hilbert spaces. In particular, in [16] an inexact-Newton method has been applied to a tomographic configuration, in which the target is assumed to be a cylindrical one and the imaging process is performed in free space and at a fixed frequency. In this paper, the above formulation is extended to multifrequency imaging, in which the use of more than one incident radiation results in an improved processing, due to the additional information included in the input data (samples of the scattered electric field at different frequencies).

The mathematical formulation of the proposed approach is described in the following with reference to transversemagnetic illumination conditions. The capabilities and limitations of the proposed extended method are evaluated by means of numerical simulations concerning targets in noisy environments for which the forward problem is solved by using the method of moments. The inverse solution is obtained, as mentioned, by using a two-loop inexact-Newton method, which has been developed in the framework of $L^{p}$ Banach spaces. In particular, after the discretization of the continuous model (based on the electric field integral equation (EFIE)), the outer loop performs a linearization of the resulting nonlinear algebraic equation, whereas the 
inner loop solves the obtained (ill-posed) linear equation in a regularized sense by using a truncated Landweber scheme in Banach spaces.

The paper is organized as follows. The mathematical formulation of the developed approach is discussed in Section 2. Section 3 reports some numerical results aimed at validating the inversion procedure. Finally, conclusions are drawn in Section 4 .

\section{Mathematical Formulation}

Let us consider the configuration shown in Figure 1. A cylindrical target, whose cross section is enclosed in an investigation domain $\mathscr{D}_{I} \subset \mathbb{R}^{2}$, is illuminated by a set of known time-harmonic, TM- $z$ electromagnetic fields characterized by angular frequencies $\omega_{1}, \ldots, \omega_{F}$. As it is well known, under such hypotheses, the scattering problem turns out to be twodimensional and scalar [4]. The resulting scattered electric field is collected in a measurement domain $\mathscr{D}_{M} \subset \mathbb{R}^{2}$.

For the sake of simplicity, in the following a single view case is described. Moreover, the $e^{j \omega t}$ dependence is omitted, assuming a frequency domain formulation. The scattered electric field in the measurement domain, $E_{s}^{\omega}(\mathbf{r}), \mathbf{r} \in \mathscr{D}_{M}$, at the angular frequency $\omega$ is related to the dielectric properties of the inspected area by the relationship [4]

$$
E_{s}^{\omega}(\mathbf{r})=G_{\mathscr{D}_{M}}^{\omega}\left(K^{\omega} \cdot x\right) E_{t}^{\omega}(\mathbf{r}), \quad \mathbf{r} \in \mathscr{D}_{M},
$$

where

$$
x(\mathbf{r})=\left[\begin{array}{c}
\epsilon_{r}(\mathbf{r})-1 \\
\sigma(\mathbf{r})
\end{array}\right]
$$

is a vector containing the relative dielectric permittivity $\epsilon_{r}$ and the electric conductivity $\sigma, K^{\omega}=\left[\begin{array}{ll}1 & -j / \omega \epsilon_{0}\end{array}\right]^{T}\left(\epsilon_{0}\right.$ is the free space dielectric permittivity and the superscript $T$ denotes the transposition operator), $E_{t}^{\omega}(\mathbf{r}), \mathbf{r} \in \mathscr{D}_{I}$, is the total electric field inside the investigation domain, and

$$
G_{\mathscr{D}_{M}}^{\omega}(\cdot)(\mathbf{r})=-\omega^{2} \mu_{0} \epsilon_{0} \int_{\mathscr{D}_{I}}(\cdot)\left(\mathbf{r}^{\prime}\right) g_{0}^{\omega}\left(\mathbf{r}, \mathbf{r}^{\prime}\right) d \mathbf{r}^{\prime},
$$

$$
\mathbf{r} \in \mathscr{D}_{M} \text {, }
$$

$g_{0}^{\omega}$ being the free space Green's function at the angular frequency $\omega[17,18]$. It is worth noting that, assuming nondispersive materials, $x$ results to be frequency independent and real-valued. The total electric field inside the investigation area is given by

$$
E_{t}^{\omega}(\mathbf{r})=E_{i}^{\omega}(\mathbf{r})+G_{\mathscr{D}_{I}}^{\omega}\left(K^{\omega} \cdot x\right) E_{t}^{\omega}(\mathbf{r}), \quad \mathbf{r} \in \mathscr{D}_{I},
$$

where $E_{i}^{\omega}$ is the known incident electric field and $G_{\mathscr{D}_{I}}^{\omega}$ is defined as

$$
G_{\mathscr{D}_{I}}^{\omega}(\cdot)(\mathbf{r})=-\omega^{2} \mu_{0} \epsilon_{0} \int_{\mathscr{D}_{I}}(\cdot)\left(\mathbf{r}^{\prime}\right) g_{0}^{\omega}\left(\mathbf{r}, \mathbf{r}^{\prime}\right) d \mathbf{r}^{\prime}
$$

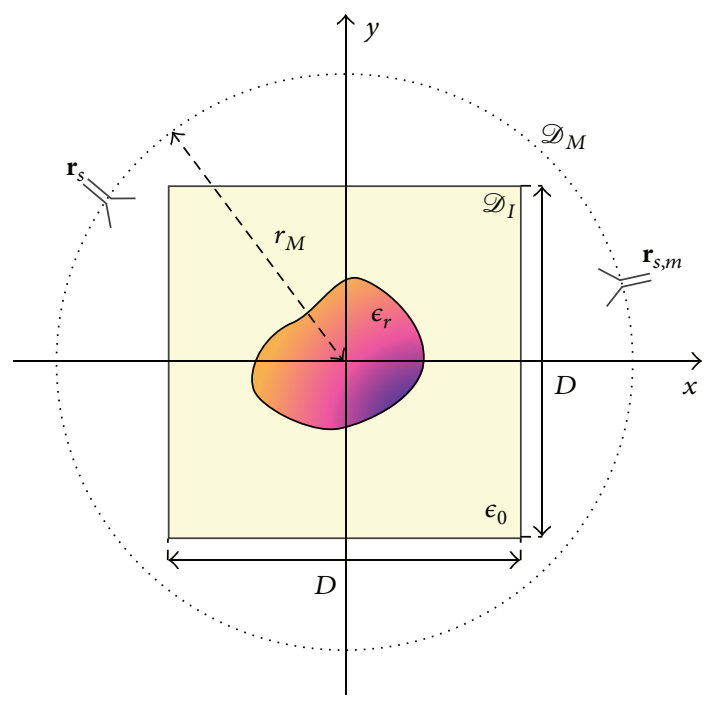

FIgURE 1: Configuration of the electromagnetic inverse scattering problem and representation of the measurement setup.

Combining (1) and (4), the scattering problem at the frequency $\omega$ can be written as

$$
E_{s}^{\omega}(\mathbf{r})=\mathscr{L}^{\omega}(x)(\mathbf{r}),
$$

where $\mathscr{L}^{\omega}$ is defined as

$$
\begin{aligned}
\mathscr{L}^{\omega} & (x)(\mathbf{r}) \\
& =G_{\mathscr{D}_{M}}^{\omega}\left(K^{\omega} \cdot x\right)\left[I-G_{\mathscr{D}_{I}}^{\omega}\left(K^{\omega} \cdot x\right)\right]^{-1} E_{i}^{\omega}(\mathbf{r}) .
\end{aligned}
$$

By applying (6) for all the available frequencies and by separating real and imaginary parts of the involved complex functions, the scattering problem can be written as the solution of the following nonlinear system of equations:

$$
F(x)=\left[\begin{array}{c}
\mathfrak{R}\left\{\mathscr{L}^{\omega_{1}}(x)\right\} \\
\mathfrak{S}\left\{\mathscr{L}^{\omega_{1}}(x)\right\} \\
\mathfrak{R}\left\{\mathscr{L}^{\omega_{2}}(x)\right\} \\
\mathfrak{I}\left\{\mathscr{L}^{\omega_{2}}(x)\right\} \\
\vdots \\
\mathfrak{R}\left\{\mathscr{L}^{\omega_{F}}(x)\right\} \\
\mathfrak{I}\left\{\mathscr{L}^{\omega_{F}}(x)\right\}
\end{array}\right]=\left[\begin{array}{c}
\mathfrak{R}\left\{E_{s}^{\omega_{1}}\right\} \\
\mathfrak{I}\left\{E_{s}^{\omega_{1}}\right\} \\
\mathfrak{R}\left\{E_{s}^{\omega_{2}}\right\} \\
\mathfrak{I}\left\{E_{s}^{\omega_{2}}\right\} \\
\vdots \\
\mathfrak{R}\left\{E_{s}^{\omega_{F}}\right\} \\
\mathfrak{J}\left\{E_{s}^{\omega_{F}}\right\}
\end{array}\right]=y,
$$

where $x \in X \in L^{p}, y \in Y \in L^{p}$, and $F: X \rightarrow Y$.

The inverse problem that must be solved in order to retrieve the dielectric properties of the targets consists in finding the unknown $x$ from the nonlinear equation (8), with $y$ being the known term. To this end, a Newton-type method [19-21] developed in the framework of Banach spaces $[15,16]$ is used. The proposed approach performs a minimization of the functional $\Psi: X \rightarrow \mathbb{R}$ given by

$$
\Psi(x)=\frac{1}{2}\|F(x)-y\|_{Y}^{2},
$$


with $\|\cdot\|_{Y}$ being the norm of the $L^{p}$ Banach space $Y$. In particular, the method contains two nested loops: the outer one performs a linearization of the scattering operator $F$ around the currently reconstructed function $x$, whereas the inner loop finds a suitable regularized solution of the resulting linearized problem by using a truncated Landweber algorithm developed in $L^{p}$ Banach spaces [15].

The reconstruction method is thus composed by the following steps (Newton iterations are denoted by the index $i$ and Landweber iterations by the index $l$ ):

(a) Start the algorithm with an initial guess $x_{0}$. If no a priori information is available, $x_{0}=0$ is assumed.

(b) Compute the Newton linearization of the operator $F$ around the current value $x_{i}$ by means of its Fréchet derivative $F_{x_{i}}: X \rightarrow Y$; that is,

$$
F_{x_{i}} \delta_{i}=y-F\left(x_{i}\right) .
$$

(c) Find a function $\delta_{i}$ by using a truncated Landweber regularization loop in Banach spaces, initialized with $\delta_{i, 0}=\bar{\delta}_{i, 0}=0$ (the symbol $\div$ indicates that the function belongs to the dual space $X^{*}$ of $X$ ):

$$
\begin{aligned}
& \bar{\delta}_{i, l+1}=\bar{\delta}_{i, l}-\rho F_{x_{i}}^{*} J^{Y}\left(F_{x_{i}} \delta_{i, l}-y+F\left(x_{i}\right)\right), \\
& \delta_{i, l+1}=J^{X^{*}}\left(\bar{\delta}_{i, l+1}\right),
\end{aligned}
$$

where $\rho>0$ is an adequate step length, $F_{x_{i}}^{*}: Y^{*} \rightarrow X^{*}$ is the adjoint operator of $F_{x_{i}}$, and the operators $J^{Y}$ and $J^{X^{*}}$ are the duality maps of the spaces $Y$ and $X^{*}$. We recall that, in $L^{p}$ Banach spaces, the duality maps are defined as

$$
J^{L^{p}}(a)=\|a\|_{L^{p}}^{2-p}|a|^{p-1} \operatorname{sign}(a)
$$

with

$$
\operatorname{sign}(a)= \begin{cases}-1 & a<0 \\ 0 & a=0 \\ 1 & a>0 .\end{cases}
$$

The Landweber steps (11) are iterated for $l=$ $0,1, \ldots, L_{\max }^{\mathrm{LW}}$.

(d) Update the solution with the increment $\delta_{i}$; that is,

$$
x_{i+1}=x_{i}+\delta_{i} .
$$

(e) Iterate Newton steps (b)-(d) for $i=0,1, \ldots, I_{\max }^{\mathrm{IN}}$, or until an a priori stopping criterion is reached.

An important remark has to be devoted to the Fréchet derivative $F_{x_{i}}$ of the operator $F$, necessary for performing steps (b) and (c). In this case, it has the following structure:

$$
F_{x_{i}}=\left[\begin{array}{c}
L_{x_{i}}^{\omega_{1}} \\
L_{x_{i}}^{\omega_{2}} \\
\vdots \\
L_{x_{i}}^{\omega_{F}}
\end{array}\right],
$$

where

$$
L_{x_{i}}^{\omega}=\left[\begin{array}{cc}
\mathfrak{R}\left\{\mathscr{L}_{x_{i}}^{\omega}\right\} & \frac{1}{\omega \epsilon_{0}} \mathfrak{I}\left\{\mathscr{L}_{x_{i}}^{\omega}\right\} \\
\mathfrak{I}\left\{\mathscr{L}_{x_{i}}^{\omega}\right\} & -\frac{1}{\omega \epsilon_{0}} \mathfrak{R}\left\{\mathscr{L}_{x_{i}}^{\omega}\right\}
\end{array}\right] .
$$

In (16), the operator $\mathscr{L}_{x_{i}}^{\omega}$ is defined as

$$
\mathscr{L}_{x_{i}}^{\omega} v=G_{x_{i}}^{\omega} v E_{x_{i}}^{\omega}
$$

with $E_{x_{i}}^{\omega}(\mathbf{r})=\left[I-G_{\mathscr{D}_{I}}^{\omega}\left(K^{\omega} \cdot x_{i}\right)\right]^{-1} E_{i}^{\omega}(\mathbf{r})$ being the electric field due to the dielectric profile $x_{i}$, and

$$
G_{x_{i}}^{\omega}(\cdot)(\mathbf{r})=-\omega^{2} \mu_{0} \epsilon_{0} \int_{\mathscr{D}_{I}}(\cdot)\left(\mathbf{r}^{\prime}\right) g_{x_{i}}^{\omega}\left(\mathbf{r}, \mathbf{r}^{\prime}\right) d \mathbf{r}^{\prime}
$$

$$
\mathbf{r} \in \mathscr{D}_{I} \text {, }
$$

where $g_{x_{i}}^{\omega}$ is the inhomogeneous Green's function related to the dielectric profile $x_{i}[4]$.

\section{Numerical Results}

The proposed multifrequency imaging method in $L^{p}$ Banach spaces has been validated by using synthetic data. In the simulations presented in this section, we assume a multiview measurement setup, in which the object is sequentially illuminated by transmitting antennas located in $S$ different positions. With reference to the configuration reported in Figure 1 , in the $s$ th view $(s=1, \ldots, S)$ the incident electric field is generated by an antenna, modeled as a line-current source, located at

$$
\mathbf{r}_{s}=\left(r_{M} \cos \psi_{s}, r_{M} \sin \psi_{s}\right)
$$

with $r_{M}=0.15 \mathrm{~m}$ and $\psi_{s}=2 \pi(s-1) / S$. For each view, the field scattered by the target is collected in $M=S-1$ measurement points on the same circumference at positions

$$
\mathbf{r}_{s, m}=\left(r_{M} \cos \psi_{m, s}, r_{M} \sin \psi_{m, s}\right),
$$

where $\psi_{m, s}=\psi_{s}+2 \pi m / S($ with $m=1, \ldots, M ; s=1, \ldots, S)$.

The scattered field data are computed by a numerical code based on the method of moments [22] and then corrupted by a Gaussian noise with zero mean value and variance corresponding to a fixed signal-to-noise ratio (SNR). The considered investigation domain is a square area of side $D=0.2 \mathrm{~m}$, centered at the origin. It has been discretized into $N_{f}=63 \times 63$ square subdomains for solving the forward electromagnetic problem and $N=40 \times 40$ cells for the inverse one. The object under test is composed of a known lossless structure (a circular cylinder of radius $r_{1}=0.075 \mathrm{~m}$, centered at the origin, with relative dielectric permittivity $\epsilon_{r}=2$ ) with three cylindrical inclusions of different shapes and dimensions. The first one is a cylinder with rectangular cross section, characterized by $\epsilon_{r}=3$, centered at $(0.04,0) \mathrm{m}$, with $x$ side and $y$ side of length $d_{x}=0.03 \mathrm{~m}$ and $d_{y}=0.06 \mathrm{~m}$, respectively. In addition, we have two circular cylinders of radii $r_{2}=0.015 \mathrm{~m}$ and $r_{3}=$ 


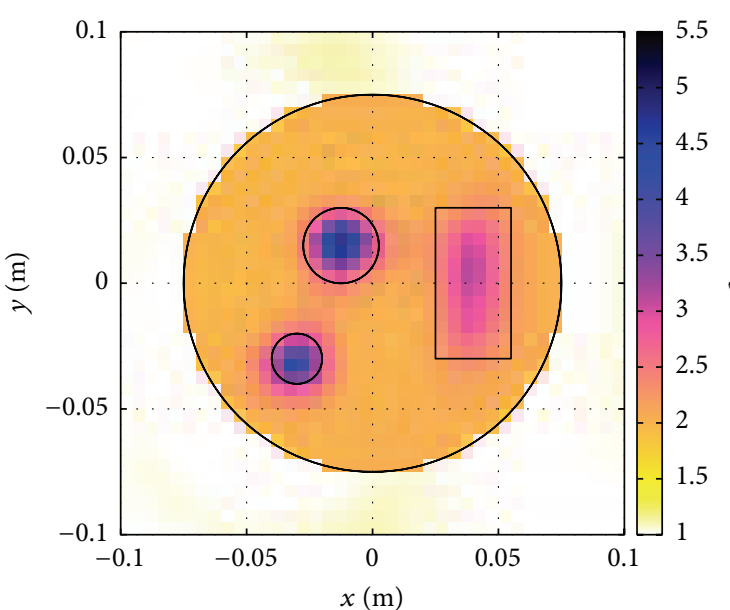

(a)

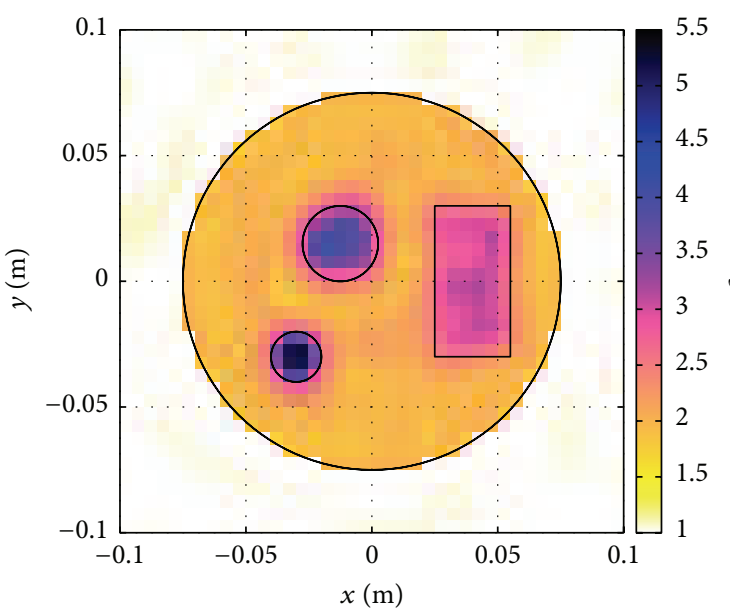

(c)

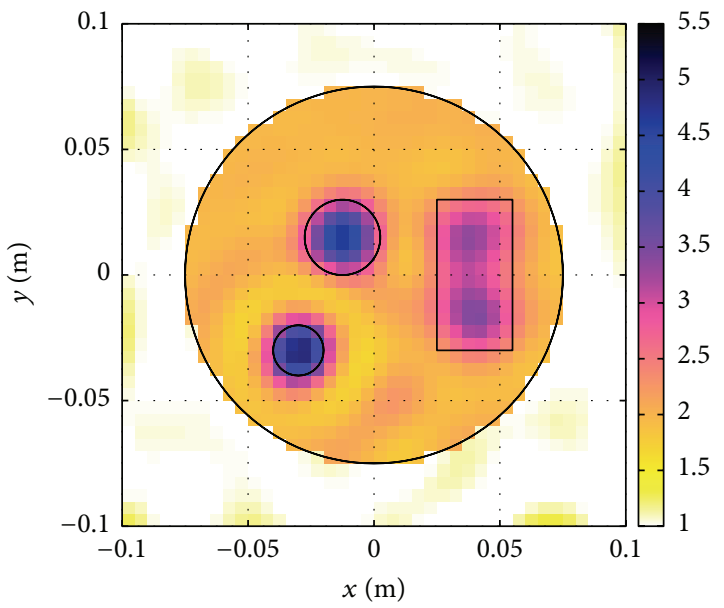

(e)

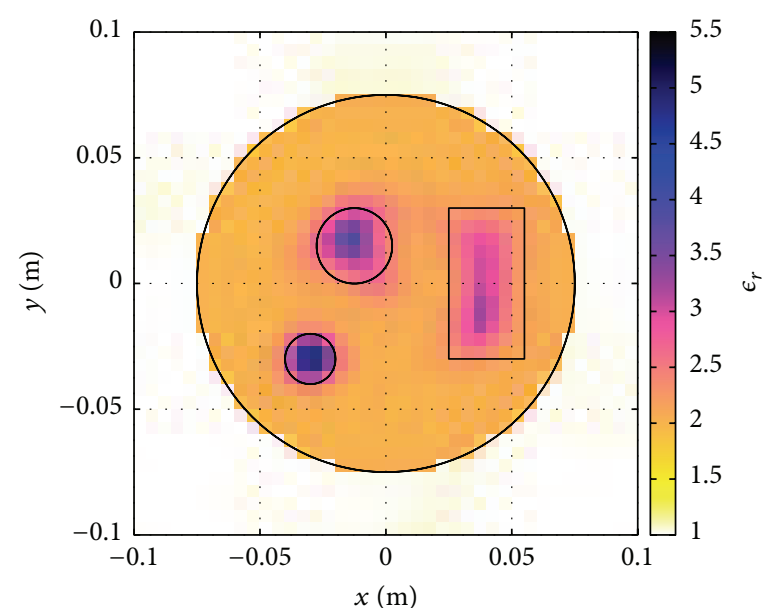

(b)

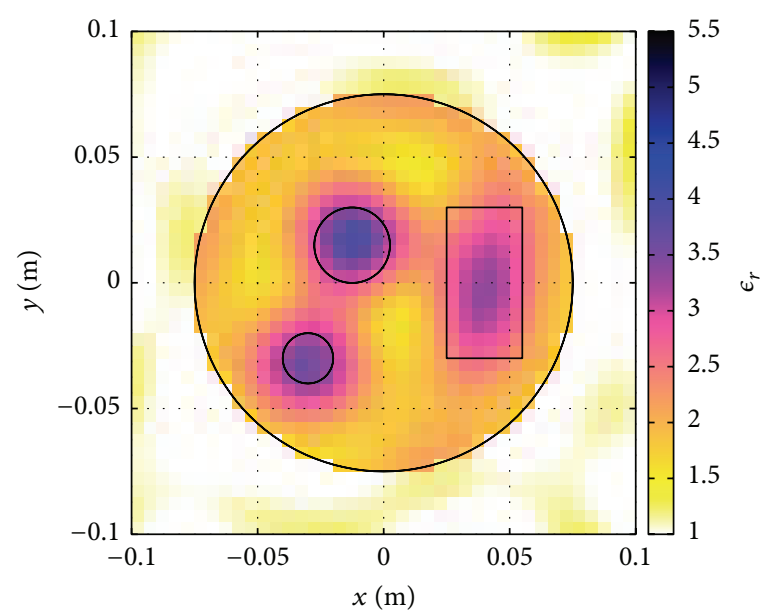

(d)

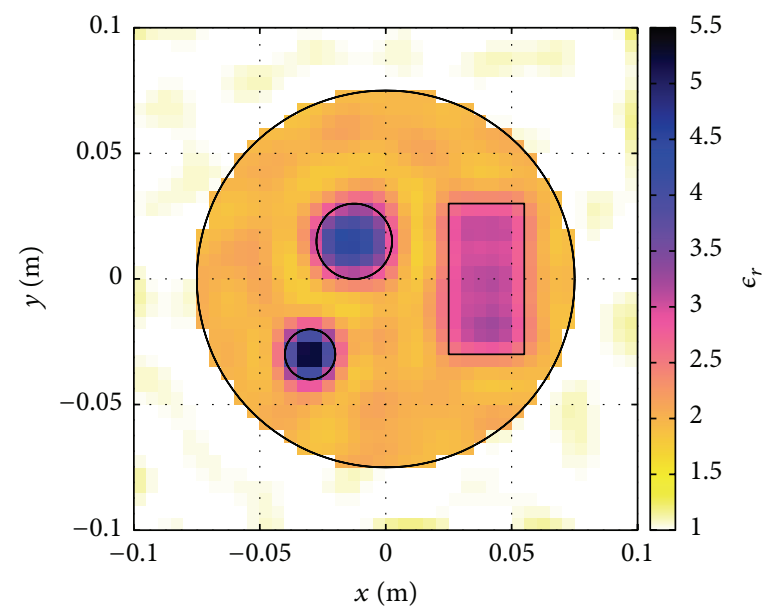

(f)

FIGURE 2: Reconstructed distributions of the relative dielectric permittivity of the investigation domain in different operating conditions, with $S=20$ and SNR $=20 \mathrm{~dB}$. Banach space reconstructions: (a) $F=1, p=1.3$; (b) $F=2, p=1.3$; (c) $F=3, p=1.6$. Hilbert space reconstructions: (d) $F=1, p=2.0$; (e) $F=2, p=2.0$; (f) $F=3, p=2.0$. 


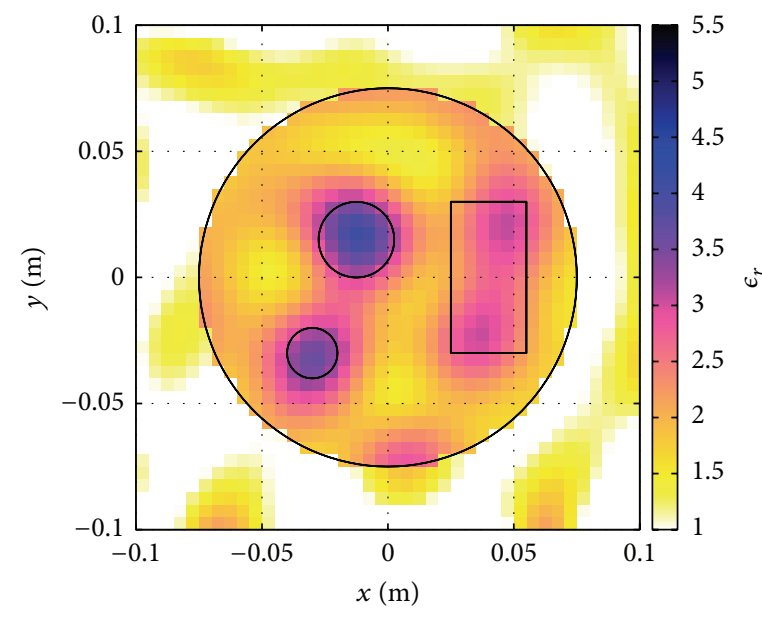

(a)

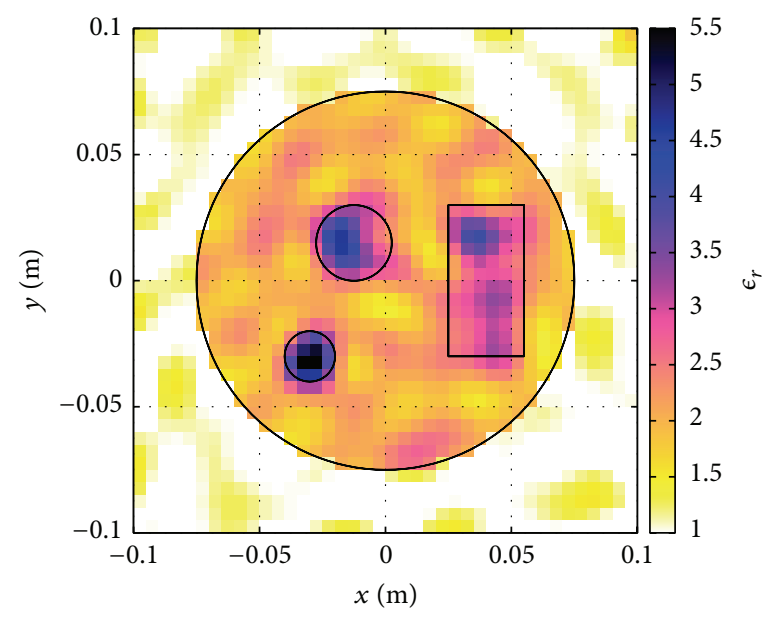

(b)

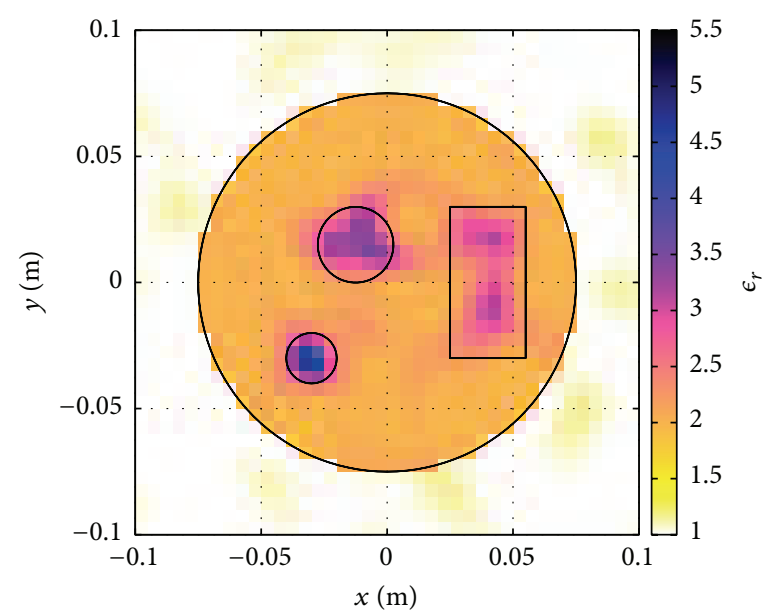

(c)

FIGURE 3: Reconstructed distributions of the relative dielectric permittivity of the investigation domain in different operating conditions, with $S=20$ and $\mathrm{SNR}=5 \mathrm{~dB}$ : (a) $F=1, p=2.0$; (b) $F=3, p=2.0$; (c) $F=3, p=1.3$.

$0.01 \mathrm{~m}$, centered at $(-0.0125,0.015) \mathrm{m}$ and $(-0.03,-0.03) \mathrm{m}$, with relative dielectric permittivities $\epsilon_{r}=4$ and $\epsilon_{r}=$ 5 , respectively. Such values have been chosen since many materials commonly used in industrial processes (e.g., plastic and ceramic compounds, wood, and concrete) have dielectric properties in this range.

The object under test has been illuminated by means of electromagnetic fields at three different frequencies: $f_{1}=$ $2 \mathrm{GHz}, f_{2}=3 \mathrm{GHz}$, and $f_{3}=4 \mathrm{GHz}$. The behavior of the inversion method has been evaluated with different numbers of frequencies used by the multifrequency reconstruction procedure $(F)$. For instance, with $F=1$ only the data at frequency $f_{1}$ is considered; for $F=2$ we use the data obtained at $f_{1}$ and $f_{2}$, and for $F=3$ all the available frequency data are used. As regards the inversion method, $L_{\max }^{\mathrm{LW}}=10$ inner Landweber iterations and $I_{\max }^{\mathrm{IN}}=20$ outer Gauss-Newton steps have been executed in all cases. The algorithm has been initialized with the outer (known) dielectric cylinder of radius $r_{1}$ and relative dielectric permittivity $\epsilon_{r}=2$ as starting guess.
In order to analyze the method's performance in different operating conditions, numerical simulations with SNR $\epsilon$ $\{5,10,15,20,15,30\} \mathrm{dB}$ and $S \in\{10,15,20,25,30\}$ have been performed. Firstly, let us consider the case with $S=20$ and SNR $=20 \mathrm{~dB}$. The results obtained by the present Banach space inversion technique are shown in Figures 2(a)-2(c).

For comparison purposes, the results provided by a standard inexact-Newton method working in Hilbert spaces [20] are also provided in Figures 2(d)-2(f). From (a) to (c) and from (d) to (f), the dielectric reconstructions obtained with one, two, and three frequencies are shown. Clearly, the improvement introduced by the Banach space approach is evident, even with a single operating frequency. An increment in the number of considered frequencies produces a further enhancement in the reconstruction quality, with both the Hilbert space and the Banach space techniques. However, as can be seen in Figure 2, the Banach space method outperforms the standard Hilbert space one in all cases. Furthermore, from a practical point of view, the Banach space results obtained with one and three frequencies are 


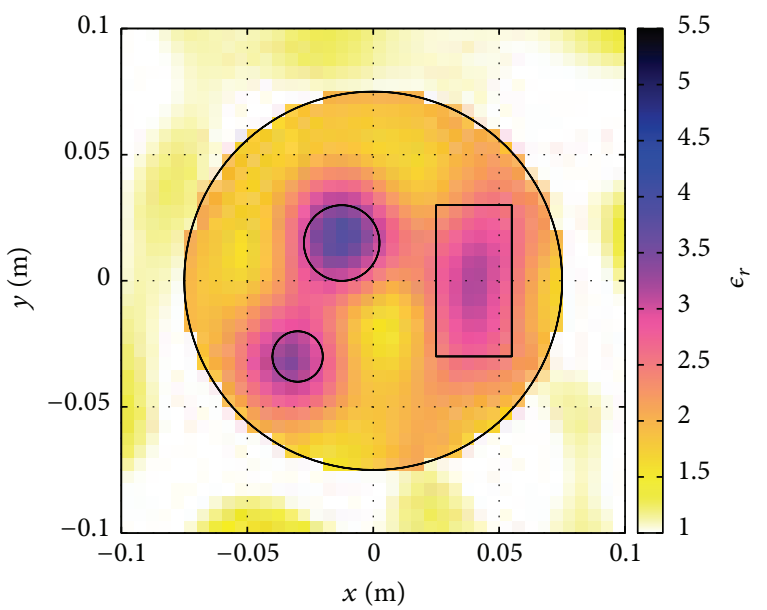

(a)

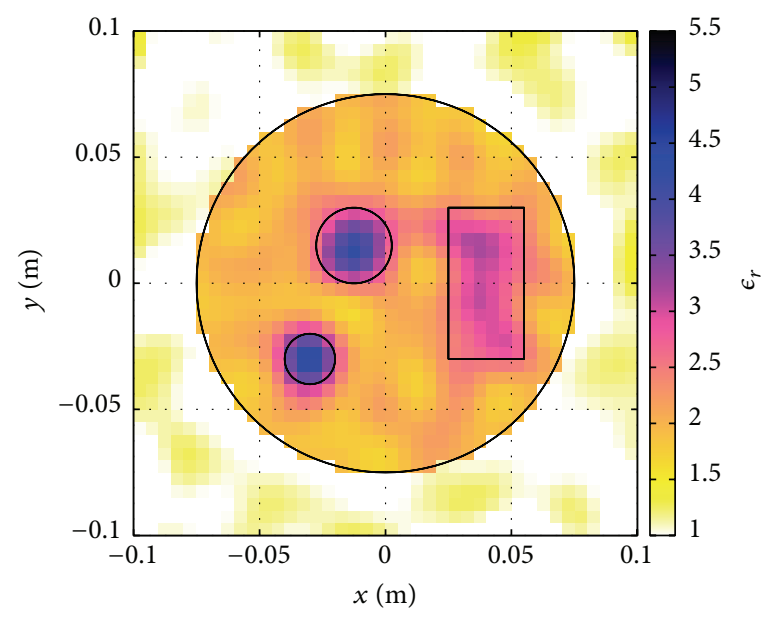

(b)

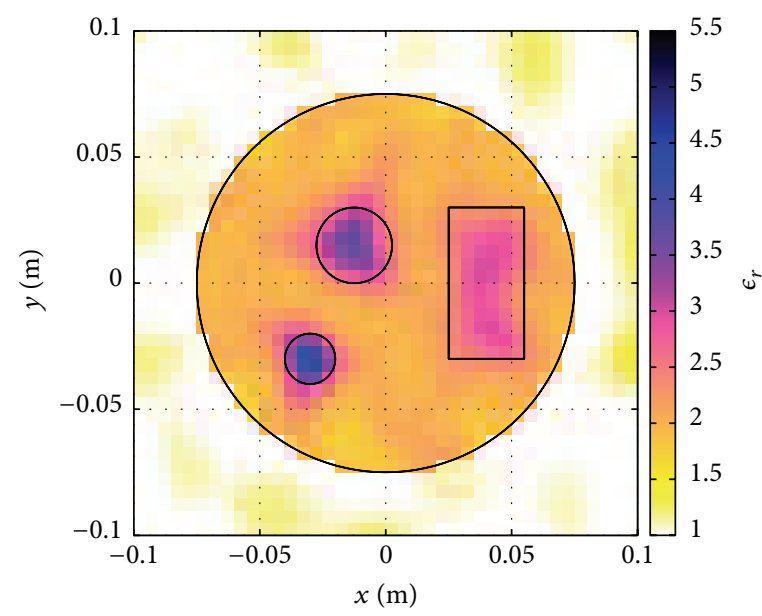

(c)

FIGURE 4: Reconstructed distributions of the relative dielectric permittivity of the investigation domain in different operating conditions, with $S=10$ and SNR $=20 \mathrm{~dB}$ : (a) $F=1, p=2.0$; (b) $F=3, p=2.0$; (c) $F=3, p=1.6$.

comparable. Therefore, we can say that in this case the proposed technique is able to provide very good results even with a reduced amount of data.

The graphs in Figure 3 are about a high-noise case, with SNR $=5 \mathrm{~dB}$. Even in this situation, the benefits of combining the Banach space regularization with the multifrequency processing are clear. In fact, the Hilbert space $(p=2)$ reconstruction with $F=3$ frequencies presented in Figure 3(b) suffers from significant ringing effects on the background and on the object area. By lowering the Banach space norm parameter $p$ to 1.3 (Figure 3(c)) it is possible to reduce these unwanted effects, improving the reconstruction performance.

In Figure 4 the inversion results with a low number of views $(S=10)$ are presented. Under these operating conditions, due to the small amount of data, the Banach space method combined with the multifrequency approach seems to be useful for reducing the reconstruction artifacts, as can be seen from the comparison of Figure 4(a) (the standard single frequency Hilbert space reconstruction) with the results in Figures 4(b) and 4(c).
The inversion results have been quantitatively evaluated using the following relative reconstruction error on the whole investigation domain

$$
e_{\text {tot }}=\frac{1}{N} \sum_{\mathbf{r}_{n} \in \mathscr{D}_{I}} \frac{\left|\epsilon_{r}\left(\mathbf{r}_{n}\right)-\overline{\epsilon_{r}}\left(\mathbf{r}_{n}\right)\right|}{\overline{\epsilon_{r}}\left(\mathbf{r}_{n}\right)}
$$

where $\overline{\epsilon_{r}}\left(\mathbf{r}_{n}\right)$ denotes the actual value of the relative dielectric permittivity in the $n$th cell $(n=1, \ldots, N)$ of the investigation domain $\mathscr{D}_{I}$ and $\epsilon_{r}\left(\mathbf{r}_{n}\right)$ is the corresponding reconstructed quantity. The trends of the reconstruction error $e_{\text {tot }}$ versus the SNR and the norm parameter $p$ are reported in Figure 5. As can be noticed, in the multifrequency results of Figures 5(b) and 5(c) the relative reconstruction error is generally lower than the single frequency case of Figure 5(a). Moreover, the effectiveness of the Banach space reconstruction approach with $p<2$ in the multifrequency case appears to be more evident in presence of low values of SNR. In Figure 6 the behavior of the reconstruction error versus the number of views $S$ and the parameter $p$ is shown. 


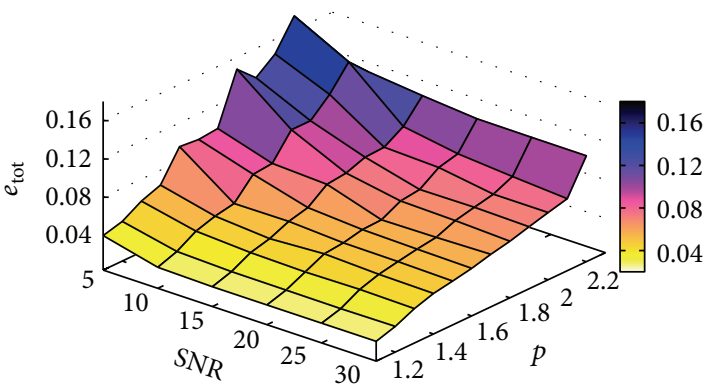

(a)

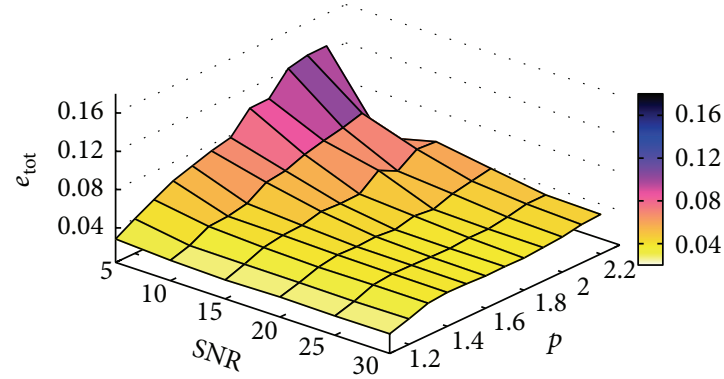

(b)

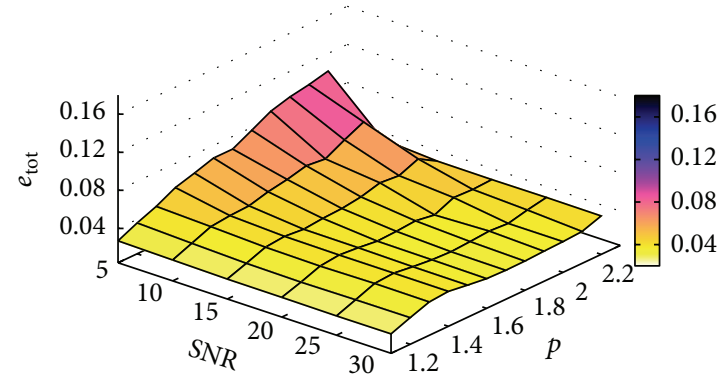

(c)

FIGURE 5: Relative reconstruction error on the investigation domain versus the $L^{p}$ Banach space norm parameter $p$ and the SNR, for $S=20$ : (a) one frequency, $F=1$; (b) two frequencies, $F=2$; (c) three frequencies, $F=3$.

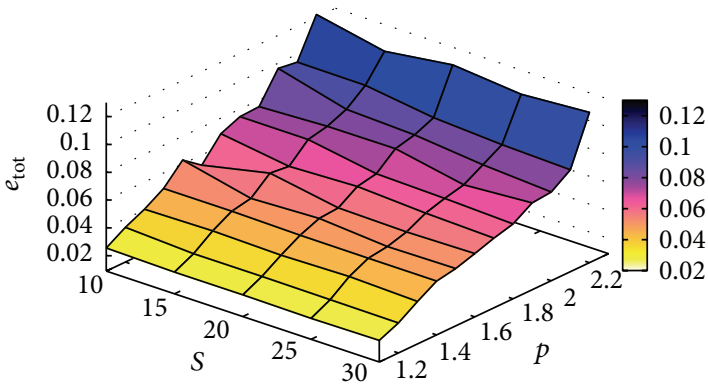

(a)

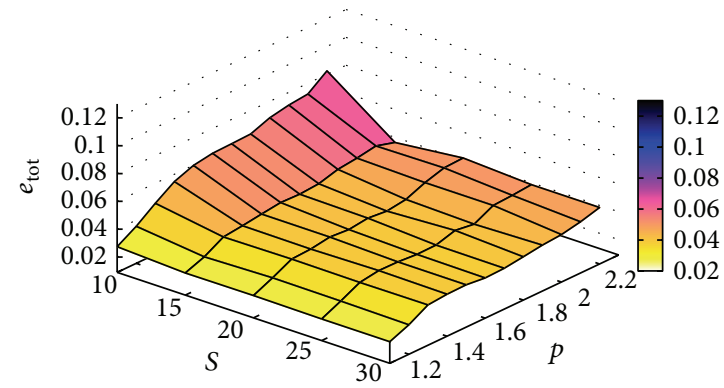

(b)

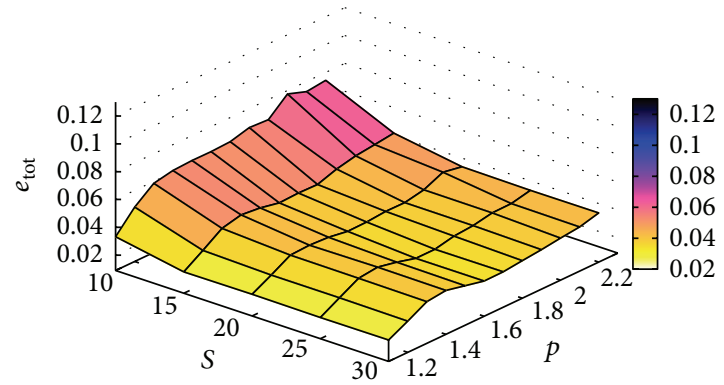

(c)

FIGURE 6: Relative reconstruction error on the investigation domain versus the $L^{p}$ Banach space norm parameter $p$ and the number of views $S$, for $\mathrm{SNR}=20 \mathrm{~dB}$ : (a) one frequency, $F=1$; (b) two frequencies, $F=2$; (c) three frequencies, $F=3$. 
In this case, too, we can observe that on one hand the reconstruction error decreases with an increase in the number of considered frequencies; on the other hand, the use of a Banach space norm leads to significant improvements especially with a reduced number of views $S$ (i.e., with a low amount of data).

\section{Conclusions}

In this paper, a previously developed approach to microwave imaging has been extended to deal with multifrequency processing in free space. The proposed technique is based on an inexact-Newton method and is aimed at inspecting inhomogeneous dielectric cylinders of arbitrary shapes under transverse-magnetic illumination conditions. The use of the inexact-Newton method (modified to take profit of the features of $L^{p}$ Banach space norms) has been found to represent a very effective regularization tool for solving the electric field integral equations considered in this paper. Numerical results have confirmed the above reconstruction capabilities of the two-loop inversion method with reference to a tomographic imaging configuration. Moreover, the advantages in terms of the reconstruction accuracy of using multifrequency imaging have been proved at least for the considered targets. Future works will be devoted to a more comprehensive numerical assessment of the method, for example, by considering different measurement setups or targets with higher dielectric contrasts. Moreover, experimental data will also be used to further validate the proposed technique in more realistic conditions.

\section{Competing Interests}

The authors declare that there are no competing interests regarding the publication of this paper.

\section{References}

[1] T. Schuster, Ed., Regularization Methods in Banach Spaces, De Gruyter, Berlin, Germany, 2012.

[2] T. Hein and K. S. Kazimierski, "Accelerated Landweber iteration in Banach spaces," Inverse Problems, vol. 26, no. 5, Article ID 055002, 2010.

[3] F. Schöpfer, A. K. Louis, and T. Schuster, "Nonlinear iterative methods for linear ill-posed problems in Banach spaces," Inverse Problems, vol. 22, no. 1, pp. 311-329, 2006.

[4] M. Pastorino, Microwave Imaging, John Wiley \& Sons, Hoboken, NJ, USA, 2010.

[5] D. Colton and R. Kress, Inverse Acoustic and Electromagnetic Scattering Theory, vol. 93, Springer, New York, NY, USA, 2013.

[6] M. R. Eskandari, R. Safian, and M. Dehmollaian, "Threedimensional near-field microwave imaging using hybrid linear sampling and level set methods in a medium with compact support," IEEE Transactions on Antennas and Propagation, vol. 62, no. 10, pp. 5117-5125, 2014.

[7] I. Catapano, A. Randazzo, E. Slob, and R. Solimene, "GPR imaging via qualitative and quantitative approaches," in Civil Engineering Applications of Ground Penetrating Radar, A. Benedetto and L. Pajewski, Eds., Springer Transactions in Civil and Environmental Engineering, pp. 239-280, Springer, Berlin, Germany, 2015.

[8] M. Fallahpour, J. T. Case, M. T. Ghasr, and R. Zoughi, "Piecewise and Wiener filter-based SAR techniques for monostatic microwave imaging of layered structures," IEEE Transactions on Antennas and Propagation, vol. 62, no. 1, pp. 282-294, 2014.

[9] M. Pastorino, M. Raffetto, and A. Randazzo, "Electromagnetic inverse scattering of axially moving cylindrical targets," IEEE Transactions on Geoscience and Remote Sensing, vol. 53, no. 3, pp. 1452-1462, 2015.

[10] W. Zhang and Q. H. Liu, "Three-dimensional scattering and inverse scattering from objects with simultaneous permittivity and permeability contrasts," IEEE Transactions on Geoscience and Remote Sensing, vol. 53, no. 1, pp. 429-439, 2015.

[11] L. Poli, G. Oliveri, F. Viani, and A. Massa, "MT-BCS-based microwave imaging approach through minimum-norm current expansion," IEEE Transactions on Antennas and Propagation, vol. 61, no. 9, pp. 4722-4732, 2013.

[12] A. Massa, M. Pastorino, and A. Randazzo, "Reconstruction of two-dimensional buried objects by a differential evolution method," Inverse Problems, vol. 20, no. 6, pp. S135-S150, 2004.

[13] M. Brignone, G. Bozza, A. Randazzo, M. Piana, and M. Pastorino, "A hybrid approach to 3D microwave imaging by using linear sampling and ACO," IEEE Transactions on Antennas and Propagation, vol. 56, no. 10, pp. 3224-3232, 2008.

[14] A. Abubakar, T. M. Habashy, G. Pan, and M.-K. Li, "Application of the multiplicative regularized Gauss-Newton algorithm for three-dimensional microwave imaging," IEEE Transactions on Antennas and Propagation, vol. 60, no. 5, pp. 2431-2441, 2012.

[15] C. Estatico, M. Pastorino, and A. Randazzo, "A novel microwave imaging approach based on regularization in $L^{p}$ Banach spaces," IEEE Transactions on Antennas and Propagation, vol. 60, no. 7, pp. 3373-3381, 2012.

[16] C. Estatico, A. Fedeli, M. Pastorino, and A. Randazzo, "Microwave imaging of elliptically shaped dielectric cylinders by means of an Lp Banach-space inversion algorithm," Measurement Science and Technology, vol. 24, no. 7, Article ID 074017, 2013.

[17] C. A. Balanis, Advanced Engineering Electromagnetics, John Wiley \& Sons, Hoboken, NJ, USA, 2nd edition, 2012.

[18] M. Pastorino, M. Raffetto, and A. Randazzo, "Two-dimensional Green's function for scattering and radiation problems in elliptically-layered media," IEEE Transactions on Antennas and Propagation, vol. 62, no. 4, pp. 2071-2080, 2014.

[19] M. Ostadrahimi, P. Mojabi, A. Zakaria, J. LoVetri, and L. Shafai, "Enhancement of Gauss-Newton inversion method for biological tissue imaging," IEEE Transactions on Microwave Theory and Techniques, vol. 61, no. 9, pp. 3424-3434, 2013.

[20] C. Estatico, G. Bozza, A. Massa, M. Pastorino, and A. Randazzo, "A two-step iterative inexact-Newton method for electromagnetic imaging of dielectric structures from real data," Inverse Problems, vol. 21, no. 6, pp. S81-S94, 2005.

[21] A. Randazzo, G. Oliveri, A. Massa, and M. Pastorino, "Electromagnetic inversion with the multiscaling inexact Newton method-experimental validation," Microwave and Optical Technology Letters, vol. 53, no. 12, pp. 2834-2838, 2011.

[22] J. Richmond, "Scattering by a dielectric cylinder of arbitrary cross section shape," IEEE Transactions on Antennas and Propagation, vol. 13, no. 3, pp. 334-341, 1965. 


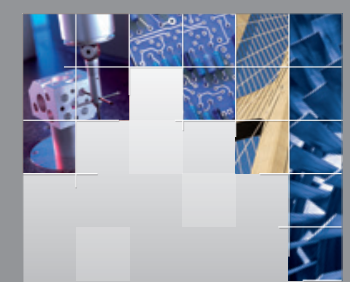

\section{Enfincering}
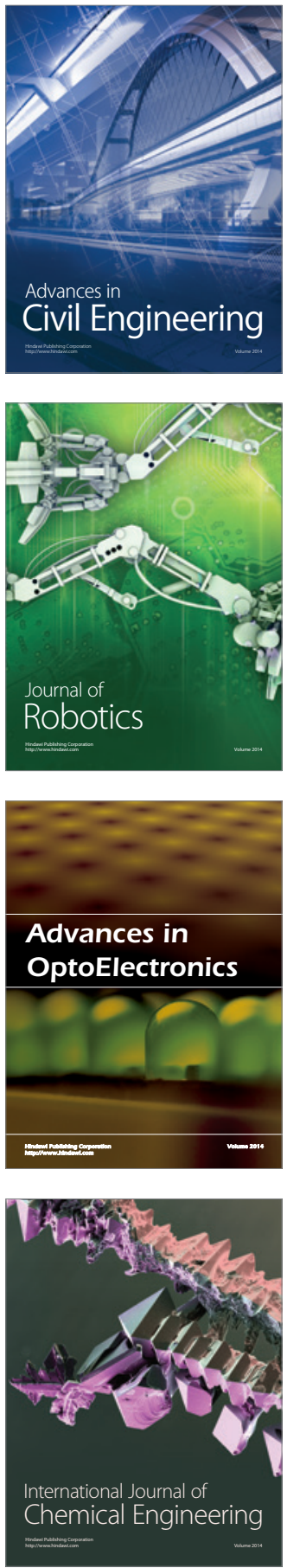

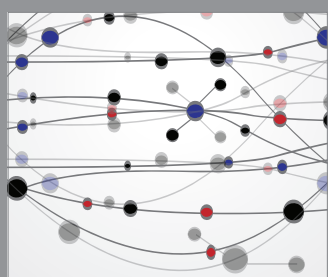

The Scientific World Journal

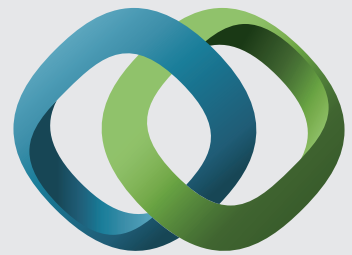

\section{Hindawi}

Submit your manuscripts at

http://www.hindawi.com
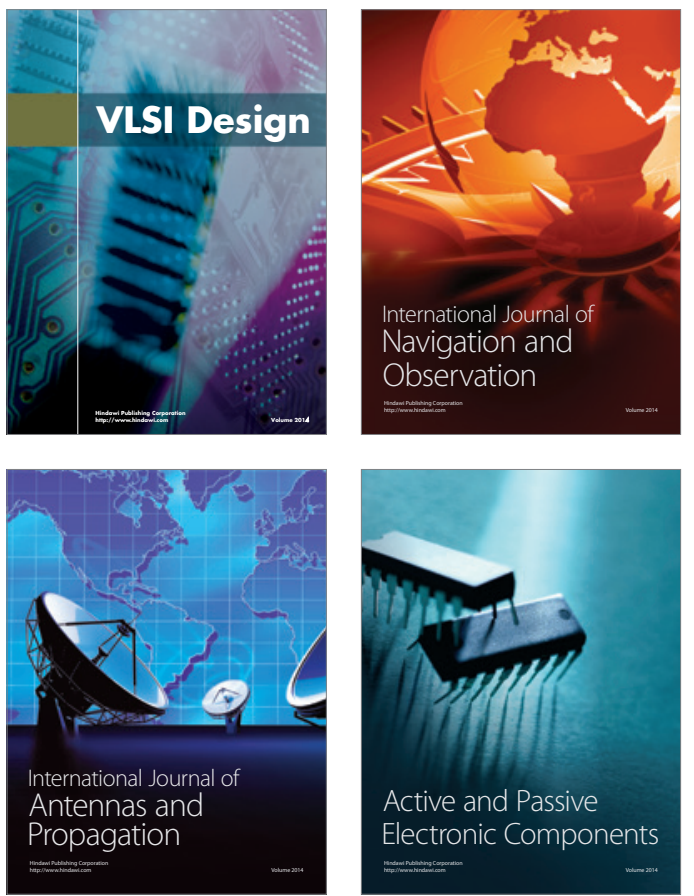
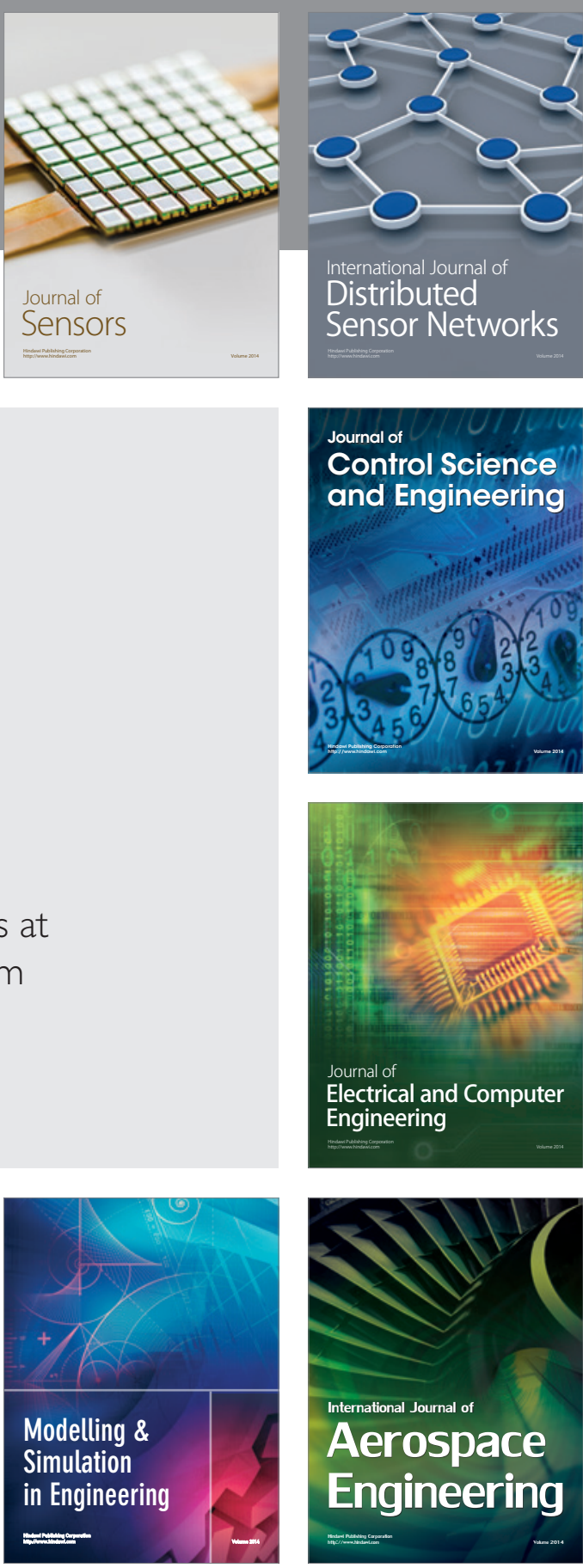

International Journal of

Distributed

Sensor Networks

Journal of

Control Science

and Engineering
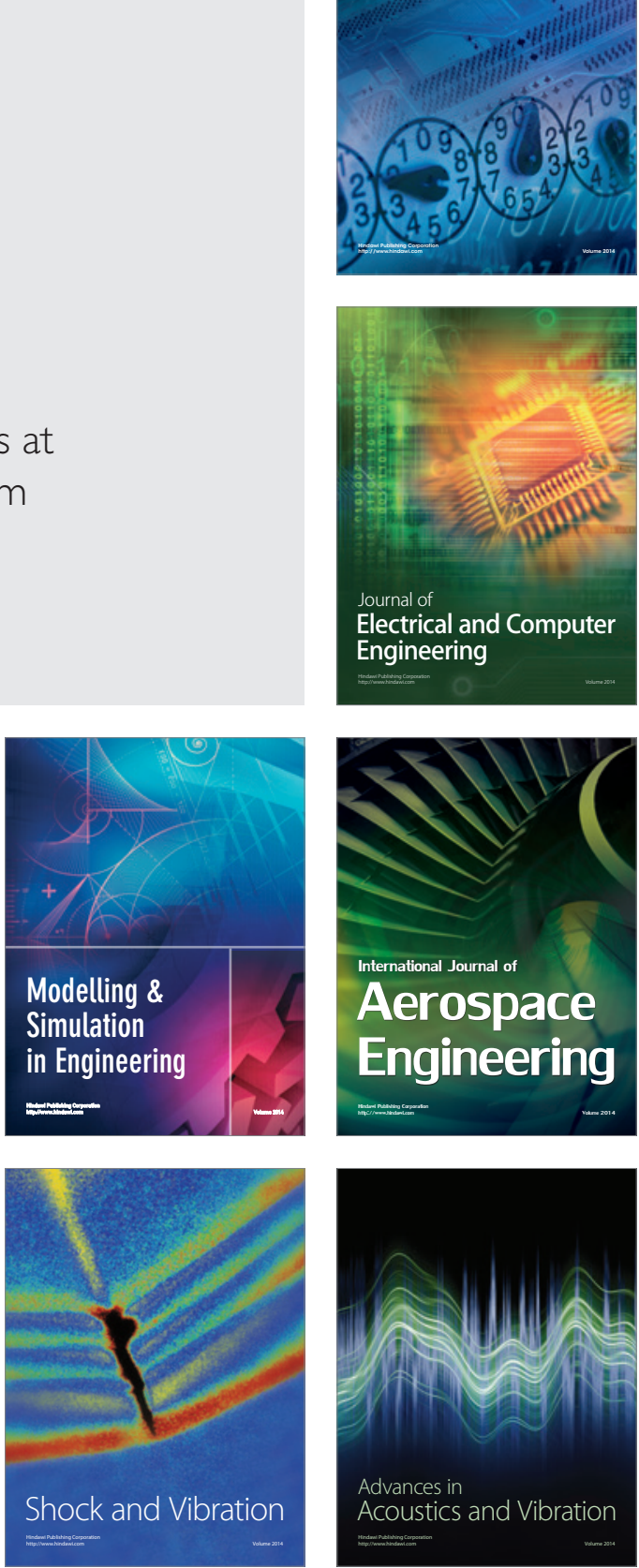